\title{
Analysis of power spectra of Doppler shift time series as a diagnostic tool for quiescent coronal loops
}

\author{
Y. Taroyan ${ }^{1}$, R. Erdélyi ${ }^{1}$, J. G. Doyle ${ }^{2}$, and S. J. Bradshaw ${ }^{3}$ \\ 1 Solar Physics and upper-Atmosphere Research Group, Department of Applied Mathematics, University of Sheffield, \\ Sheffield S3 7RH, UK \\ e-mail: Y.Taroyan@sheffield.ac.uk \\ 2 Armagh Observatory, College Hill, Armagh BT61 9DG, N. Ireland \\ 3 Space \& Atmospheric Physics, Blackett Laboratory, Imperial College London, Prince Consort Road, \\ London SW7 2BZ, UK
}

Received 19 July 2006 / Accepted 24 October 2006

\section{ABSTRACT}

\begin{abstract}
Context. Analysing the structure of solar coronal loops is crucial to our understanding of the processes which heat and maintain the coronal plasma at multimillion degree temperatures. The determination of the physical parameters of the loops remains both an observational and theoretical challenge.

Aims. The present work is aimed at tackling some of these challenges such as the determination of the average loop temperature and its distribution along a given loop.

Methods. A novel diagnostic technique for quiescent coronal loops based on the analysis of power spectra of Doppler shift time series is proposed. It is assumed that the loop is heated randomly both in space and time by small-scale discrete impulsive events of unspecified nature. The loop evolution is characterised by longitudinal motions caused by the random heating events. These random motions can be represented as a superposition of the normal modes of the loop, i.e., its standing acoustic wave harmonics. The idea is borrowed from helioseismology where a similar approach resulted in a deep understanding of the solar interior.

Results. It is shown that depending on the heliographic position of the loop and its geometrical orientation, various harmonics can be identified in the power spectra of the line shift time series. The highest power peak corresponds to the fundamental mode. The peaks become smaller as the frequency increases. The frequencies of the harmonics are determined by the loop length and temperature and thus are suggested to be used as a temperature diagnostic tool. It is demonstrated that the analysis of the power spectra allows the distinction between uniformly heated loops from loops heated near their footpoints and to estimate the average energy of a single heating event. The proposed new method could in principle be used to study the multithermal structure of coronal loops.

Conclusions. The power spectrum analysis is a potentially powerful technique for coronal loop diagnostics.
\end{abstract}

Key words. Sun: corona - Sun: oscillations - line: profiles - hydrodynamics - methods: analytical - methods: numerical

\section{Introduction}

Our understanding of the solar corona has significantly improved with the launch of the Solar and Heliospheric Observatory (SOHO) in 1995 and the Transition Region and Coronal Explorer (TRACE) in 1998. Despite this progress, however, there are still important processes in the corona that remain elusive. Primary among them is the coronal heating problem, the precise physical mechanism by which the solar atmosphere is heated to its multimillion degree temperatures. It is not yet known how this energy is stored, released and dissipated. It is, however, clear that the magnetic field plays a central role in this process. The largest contribution to X-ray emission comes from magnetic loops known as the basic building blocks of the solar atmosphere (Rosner et al. 1978). TRACE images show the complex multistrand structure of these loops. It has been recognised that the study of magnetic loops is crucial to our understanding of the dynamics of solar coronal plasma. A vast number of new studies have attempted to compare theoretical models with loop observations. Such comparisons have been carried out mostly by measuring the temperature and density profiles using line intensity ratios from spectrometers (Brković et al. 2002; Landi \& Landini 2004; Ugarte-Urra et al. 2005), differential emission measure analysis (Schmelz et al. 2002; Martens et al. 2002) or filter ratios from imagers. A successful comparison of measured and simulated temperature profiles could in principle be a diagnostic tool for loop heating. However, temperature measurements have been the subject of a heated debate. Some authors have questioned the use of these methods (see, e.g., Testa et al. 2002; Chae et al. 2002; Del Zanna \& Mason 2003; Landi \& Landini 2005; Schmelz \& Martens 2006). The measurements are often ambiguous and contradictory. A wellknown example illustrating the existing controversy in the field is a single dataset which has been interpreted in terms of uniform (Priest et al. 1998), footpoint (Aschwanden 2001) and apex (Reale 2002) heating.

In this respect, the newly emerging field of coronal seismology has a potential which has not yet been fully exploited. In solar-terrestrial physics, the word "seismology" has been traditionally associated with the solar interior. The task of global and local helioseismology is probing the invisible interior of the Sun by measuring the intensity fluctuations or the Doppler shifting of the light emitted at the solar surface. The important discoveries and achievements of helioseismology are well-known. The field of seismology of the solar magnetic atmosphere from the photosphere to the corona is still in its infancy. Despite the 
considerable success of coronal seismology over the past few years, the main efforts have been directed towards the identification and the study of the properties of different classes of waves in coronal structures. The current state of the field is reviewed by De Moortel (2005), Nakariakov \& Verwichte (2005). A major factor inhibiting the development of coronal seismology is the difficulty with which the waves are detected: firstly, most of the waves are transient and localised in nature; secondly, the temporal and spatial resolution of current space borne and ground based instruments is still not adequate enough. It is therefore not surprising that the degree of precision and the methods employed in coronal seismology remain basic compared with their counterparts in helioseismology. Future missions such as Solar-B (due for launch in 2006), Solar Dynamics Observatory (SDO) and others will carry onboard both spectrometers and imagers and will be able to make high cadence feature tracking-mode observations of quiescent coronal loops for long periods of time. The power of having simultaneous imaging and spectral data was recently shown by Doyle et al. (2006b).

The study of waves as possible heating candidates through resonant absorption, phase mixing, ion-cyclotron resonance, non-linear conversion of Alfvén waves, etc., is important in its own right. Another major candidate is the nanoflare heating mechanism proposed by Parker (1988): the loops are wound and wrapped around each other due to the continuous shuffling of their photospheric footpoints. This leads to many small and localised bursts of energy through magnetic reconnection. Several studies have concentrated on the observational consequences of such heating scenarios. The results usually indicate that both wave and reconnection driven heating appear in small scale bursts of energy which are randomly distributed along the loop (see, e.g., Moriyasu et al. 2004; Einaudi \& Velli 1999). Here we are mainly interested in studying the observational consequences of such localised heating events without specifying the exact nature of the actual heating process.

Wang et al. (2002) observed longitudinal fundamental mode standing waves in hot active region loops. Taroyan et al. (2005) and Wang et al. (2005) explained the excitation of such waves in terms of a single heating pulse at the loop footpoint. De Pontieu et al. (2004, 2005) showed that longitudinal waves continuously driven at the photospheric footpoints of the loops can tunnel and propagate into the corona providing an explanation for the upward propagating slow waves reported by De Moortel et al. (2000). Similarly, the excitation of fast kink waves has been studied both in the context of an interaction of a loop system with flare generated fast waves (Aschwanden et al. 1999; Nakariakov et al. 1999; Ruderman \& Roberts 2002) and in the context of a footpoint driver (Musielak \& Ulmschneider 2001) and the repetitive reconnection (seen as a sequence of transition region explosive events) triggered by transverse oscillations (Doyle et al. 2006a).

The possibility of wave excitation by pulses randomly distributed along a given loop has received much less attention. Taroyan et al. (2006) and Mendoza-Briceño \& Erdélyi (2006) have carried out some initial studies. Taroyan et al. (2006) also found that the nanoflare heating model is able to qualitatively reproduce the observed dynamics and the average red-shifts typical for the lines covering the upper transition region and lower corona. In the present paper, we are going to look into the possibility of applying the helioseismic approach of power spectrum analysis to coronal loops: Doppler shift time series are Fourier analysed and the resulting power spectra are examined for the presence of discrete peaks corresponding to standing wave harmonics. This could be interpreted as a manifestation of the superposition principle, i.e., any linear perturbation of a physical system is a superposition of its normal modes. However, the problem is more complicated. It is tackled both analytically and numerically. The paper mainly addresses the feasibility and the efficiency of the proposed new method.

\section{Analytical approach}

The idea we put forward can be elucidated using a simple analytical model. The model assumes that the 1D loop is in isothermal equilibrium. Small amplitude longitudinal motions inside the loop are governed by the following linear inhomogeneous equation:

$\frac{\partial^{2} v}{\partial t^{2}}-c_{\mathrm{s}}^{2} \frac{\partial^{2} v}{\partial s^{2}}=-\frac{\gamma-1}{\rho_{0}} \frac{\partial \mathcal{H}}{\partial s}, \quad 0<s<L, \quad 0<t$,

where $L$ is the length of the loop, $v=v(s, t)$ is the longitudinal velocity and $c_{\mathrm{s}}, \rho_{0}$ are the constant sound speed and density along the loop. The right-hand side of Eq. (1) represents the source term and the function $\mathcal{H}=\mathcal{H}(s, t)$ is the heating rate which needs to be specified. Equation (1) is supplied with the boundary conditions

$v(0, t)=v(L, t)=0, \quad 0<t$,

and the initial conditions

$v(s, 0)=\frac{\partial v(s, 0)}{\partial t}=0, \quad 0<s<L$.

The boundary conditions (2) are a mathematical manifestation of the effects of the dense chromosphere on small amplitude motions generated within the corona. The initial conditions (3) assume that the loop plasma is initially at rest. The problem (1)-(3) will be solved for two different source terms representing random heating.

\subsection{The Green's function}

The solution to the inhomogeneous wave Eq. (1) can be expressed in terms of the Green's function, boundary conditions (2) and initial conditions (3). The Green's function $G=G(s, t ; \xi, \tau)$ is the solution to the equation

$\frac{\partial^{2} G}{\partial t^{2}}-c_{\mathrm{s}}^{2} \frac{\partial^{2} G}{\partial s^{2}}=\delta(s-\xi) \delta(t-\tau), \quad 0<s, \xi<L, \quad 0<t, \tau$,

subject to the boundary conditions (2) and the initial conditions (3). Here $\delta$ is the Dirac delta function. The Green's function of this problem can be constructed using the method of eigenfunction expansion (see, e.g., Duffy 2001). The result is

$\begin{aligned} G(s, t ; \xi, \tau)=\sum_{k=1}^{\infty} & \frac{2}{k \pi c_{\mathrm{s}}} \sin \left(\frac{\omega_{k} \xi}{c_{\mathrm{s}}}\right) \sin \left(\frac{\omega_{k} \mathrm{~s}}{c_{\mathrm{s}}}\right) \\ & \times \sin \left[\omega_{k}(t-\tau)\right] H(t-\tau),\end{aligned}$

where $H$ denotes the Heaviside step function and

$\omega_{k}=\frac{\pi c_{\mathrm{s}} k}{L}, \quad k=1,2 \ldots$

represent the frequencies of standing wave harmonics. For a given source term the solution to the problem (1)-(3) can be expressed by the formula

$v(s, t)=-\frac{\gamma-1}{\rho_{0}} \int_{0}^{t} \int_{0}^{L} \frac{\partial \mathcal{H}(\xi, \tau)}{\partial \xi} G(s, t ; \xi, \tau) \mathrm{d} \xi \mathrm{d} \tau$. 


\subsection{Random heating by Dirac delta pulses}

We start with the consideration of the case when the loop is heated by instantaneous $\delta$ pulses which occur randomly in time and are randomly distributed along the loop. The heating function is expressed in the form

$\mathcal{H}(s, t)=\mathcal{H}_{0} \sum_{j=1}^{n} \delta\left(t-t_{j}\right) H\left(s-s_{j}+s_{\mathrm{h}}\right) H\left(s_{j}+s_{\mathrm{h}}-s\right)$,

where $n$ is the total number of pulses, $\mathcal{H}_{0}$ is the maximum heat input, $s_{j}, t_{j}$ are the random location and the random offset time of the $j$ th pulse, $s_{\mathrm{h}}$ characterises the heating scale length. It is assumed that $s_{\mathrm{h}}<s_{j}<L-s_{\mathrm{h}}$, i.e., the heating lies within the interval $(0, L)$ and has a step function profile with a $2 s_{\mathrm{h}}$ width centred at $s_{j}$. The source term on the right-hand side of Eq. (1) takes the form

$-\frac{\gamma-1}{\rho_{0}} \mathcal{H}_{0} \sum_{j=1}^{n} \delta\left(t-t_{j}\right)\left[\delta\left(s-s_{j}+s_{\mathrm{h}}\right)-\delta\left(s-s_{j}-s_{\mathrm{h}}\right)\right]$.

Expression (9) is derived using the well-known fact that the derivative of the Heaviside function is the Dirac delta function. The solution $v=v(s, t)$ is readily found using Eq. (7):

$$
\begin{array}{r}
v(s, t)=\sum_{j=1}^{n} \sum_{k=1}^{\infty} \frac{4(\gamma-1) \mathcal{H}_{0}}{\rho_{0} \pi c_{\mathrm{s}} k} \sin \left(\frac{\omega_{k} s_{\mathrm{h}}}{c_{\mathrm{s}}}\right) \cos \left(\frac{\omega_{k} s_{j}}{c_{\mathrm{s}}}\right) \\
\times \sin \left(\frac{\omega_{k} s}{c_{\mathrm{s}}}\right) \sin \left[\omega_{k}\left(t-t_{j}\right)\right] H\left(t-t_{j}\right),
\end{array}
$$

where the frequencies $\omega_{k}$ are given by Eq. (6). In the limit of vanishing heating scale length $\left(s_{\mathrm{h}} \rightarrow 0\right)$ the perturbation amplitude tends to zero.

Equation (10) shows that at each moment of time, the motion represents a superposition of standing wave harmonics $\propto \sin \left[\omega_{k}\left(t-t_{j}\right)\right] \sin \left(\omega_{k} s / c_{\mathrm{s}}\right)$. However, the coefficients of the harmonics in Eq. (10) are time-dependent Heaviside functions. In order to see the structure of the power spectrum, we Fourier analyse the velocity signal $v(s, t)$ :

$\tilde{v}(s, \omega)=\int_{0}^{t_{\mathrm{d}}} v(s, t) \exp (\mathrm{i} \omega t) \mathrm{d} t$,

where $t_{\mathrm{d}}$ is the total duration of the observations. After some algebra the following expression is obtained:

$$
\begin{aligned}
\tilde{v}(s, \omega)=\frac{4(\gamma-1) \mathcal{H}_{0}}{\rho_{0} \pi c_{\mathrm{s}}} \\
\quad \times \sum_{j=1}^{n} \sum_{k} \frac{C_{j k}}{\omega-\omega_{k}} \sin \left[\frac{\left(\omega-\omega_{k}\right)\left(t_{\mathrm{d}}-t_{j}\right)}{2}\right],
\end{aligned}
$$

where $k= \pm 1, \pm 2, \ldots$ The terms in Eq. (12) have their peaks at the frequencies of the standing waves $\omega=\omega_{k}$. The factors $t_{\mathrm{d}}-t_{j}$ influence the narrowness and height of the peaks. The coefficients $C_{j k} \propto 1 / k$ (see Appendix A) which means that the peaks become shorter with increasing harmonic number $k$.

\subsection{Random heating by finite duration pulses}

Pulses with finite duration can be characterised by a heating function of the following form:

$\mathcal{H}(s, t)=\mathcal{H}_{0} \sum_{j=1}^{n} H\left(t-t_{j}\right) H\left(t_{j}+\tau_{j}-t\right) H\left(s-s_{j}+s_{\mathrm{h}}\right) H\left(s_{j}+s_{\mathrm{h}}-s\right)$, where $\tau_{j}$ is the duration of the $j$ th pulse. Other parameters are the same as in Eq. (8). Combining Eqs. (5), (7) and (13) we have

$$
\begin{gathered}
v(s, t)=\sum_{j=1}^{n} \sum_{k=1}^{\infty} \frac{4(\gamma-1) \mathcal{H}_{0} L}{\rho_{0}\left(\pi c_{\mathrm{S}} k\right)^{2}} \sin \left(\frac{\omega_{k} s_{\mathrm{h}}}{c_{\mathrm{S}}}\right) \cos \left(\frac{\omega_{k} s_{j}}{c_{\mathrm{S}}}\right) \\
\times \sin \left(\frac{\omega_{k} s}{c_{\mathrm{s}}}\right) H\left(t-t_{j}\right)\left\{H\left(t-t_{j}-\tau_{j}\right) \cos \left[\omega_{k}\left(t-t_{j}-\tau_{j}\right)\right]\right. \\
\left.+H\left(\tau_{j}+t_{j}-t\right)-\cos \left[\omega_{k}\left(t-t_{j}\right)\right]\right\} .
\end{gathered}
$$

The expression inside the braces in Eq. (14) vanishes when the pulse duration $\tau_{j} \rightarrow 0$. Apart from the terms corresponding to standing wave harmonics, the right-hand side of Eq. (14) contains time-dependent coefficients in the form of Heaviside functions. The contribution of these coefficients to the power spectrum can be examined using the Fourier transform (11). The resulting expression has the form

$$
\begin{aligned}
& \tilde{v}(s, \omega)= \frac{4(\gamma-1) \mathcal{H}_{0} L}{\rho_{0}\left(\pi c_{\mathrm{s}}\right)^{2}} \\
& \times \sum_{j=1}^{n} \sum_{k}\left[\frac{C_{1 j k}}{\omega} \sin \left(\frac{\omega \tau_{j}}{2}\right)+\frac{C_{2 j k}}{\omega-\omega_{k}} \sin \left(\frac{\left(\omega-\omega_{k}\right)\left(t_{\mathrm{d}}-t_{j}\right)}{2}\right)\right. \\
&\left.+\frac{C_{3 j k}}{\omega-\omega_{k}} \sin \left(\frac{\left(\omega-\omega_{k}\right)\left(t_{\mathrm{d}}-t_{j}-\tau_{j}\right)}{2}\right)\right]
\end{aligned}
$$

where $k= \pm 1, \pm 2, \ldots$ The terms in Eq. (15) have their peaks at the frequencies of the standing waves $\omega=\omega_{k}$. Note the appearance of a new peak at $\omega=0$ which is due to the finite duration of the pulses. The coefficients $C_{l j k} \propto 1 / k^{2}, l=1,2,3$ (see Appendix A). Therefore, the peak decrease with increasing $k$ occurs faster than in the case of delta pulses.

\section{Numerical approach}

\subsection{Initial setup}

The initial analytical results of the preceding section show that the response of a loop to random pulses is a superposition of its standing modes. These results are valid in the limit of linear ideal MHD. In the present section, we are going to examine the loop behaviour in a more realistic situation in which losses, nonlinearity, gravitational and thermal stratification are taken into account. A confirmation of the results presented in the preceding section is not guaranteed.

The plasma motion along a loop is governed by the following set of nonlinear differential equations:

$$
\begin{aligned}
& \frac{\partial \rho}{\partial t}+\frac{\partial}{\partial s}[\rho v]=0, \\
& \frac{\partial}{\partial t}[\rho v]+\frac{\partial}{\partial s}\left[\rho v^{2}\right]=-\frac{\partial p}{\partial s}+\rho g_{\|}, \\
& \frac{\partial e}{\partial t}+\frac{\partial}{\partial s}[(e+p) v]=\rho v g_{\|}+\mathcal{S},
\end{aligned}
$$

where $s$ is the coordinate along the loop, $\rho$ is density, $p$ is pressure, $v$ is velocity,

$e=\frac{p}{\gamma-1}+\frac{\rho v^{2}}{2}$,

is the energy density,

$g_{\|}=-g_{\odot} \cos \left(\frac{\pi s}{L}\right)$ 

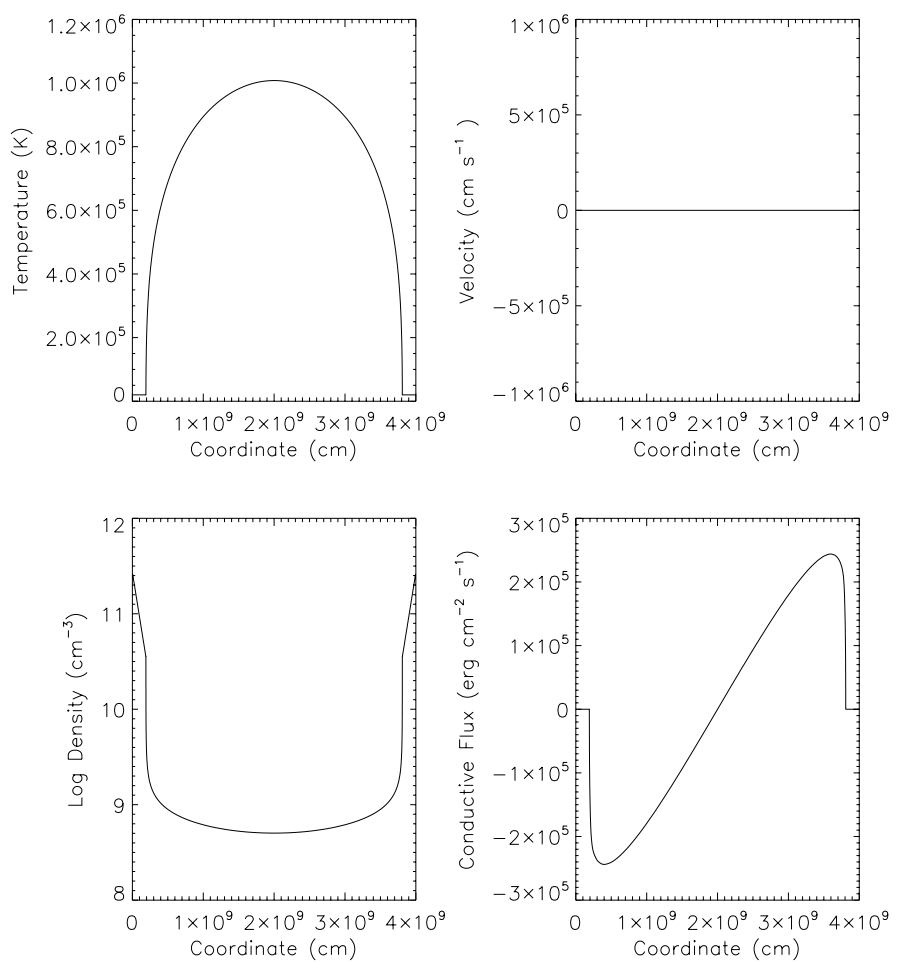

Fig. 1. A $40 \mathrm{Mm}$ long loop with an initial temperature of $T=1 \times 10^{6} \mathrm{~K}$ is in hydrostatic equilibrium.

is the component of gravitational acceleration along a semicircular loop of length $L$, and $\mathcal{S}$ represents the net effect of sources and sinks of energy. The initial loop profile is shown in Fig. 1. The loop is in hydrostatic equilibrium. It has an apex temperature of $1 \mathrm{MK}$ and the footpoints are kept at a constant chromospheric temperature of $2 \times 10^{4} \mathrm{~K}$. The balance between sources and sinks of energy is achieved by applying uniform heating of $\mathcal{H}=2.6 \times 10^{-4} \mathrm{erg} \mathrm{cm}^{-3} \mathrm{~s}^{-1}$ along the loop. The hydrostatic equation of energy has the form

$\mathcal{S} \equiv \mathcal{H}-\frac{\partial F_{\mathrm{c}}}{\partial s}-\mathcal{L}=0$,

where thermal conduction is expressed in terms of the conductive flux $F_{\mathrm{c}}$ :

$F_{\mathrm{c}}=-\varkappa T^{5 / 2} \frac{\partial T}{\partial s}$

with $\varkappa=10^{-6} \mathrm{erg} \mathrm{s}^{-1} \mathrm{~K}^{-1} \mathrm{~cm}^{-1}$ being the coefficient of thermal conduction along the magnetic field. The last term in Eq. (19) $\mathcal{L}=n^{2} \Lambda(T)$ corresponds to optically thin radiative losses: $n$ is the number density and $\Lambda=\Lambda(T)$ is the radiative loss function. Figure 1 also shows that the gradient of conductive flux, i.e., thermal conduction can have both positive and negative values along the loop. It becomes positive in the lower transition region to balance losses due to strong radiation.

\subsection{Evolution of the loop subject to random heating}

The evolution of a $40 \mathrm{Mm}$ long loop with an initial profile shown in Fig. 1 is examined by integrating the governing Eqs. (16) from $t=0 \mathrm{~s}$ to $t=20000 \mathrm{~s}$. The integration is performed using a hydrodynamic-radiative code called HYDRAD (Bradshaw \& Mason Bradshaw). An adaptive regridding scheme is used in order to ensure that the small-scale dynamic features along the
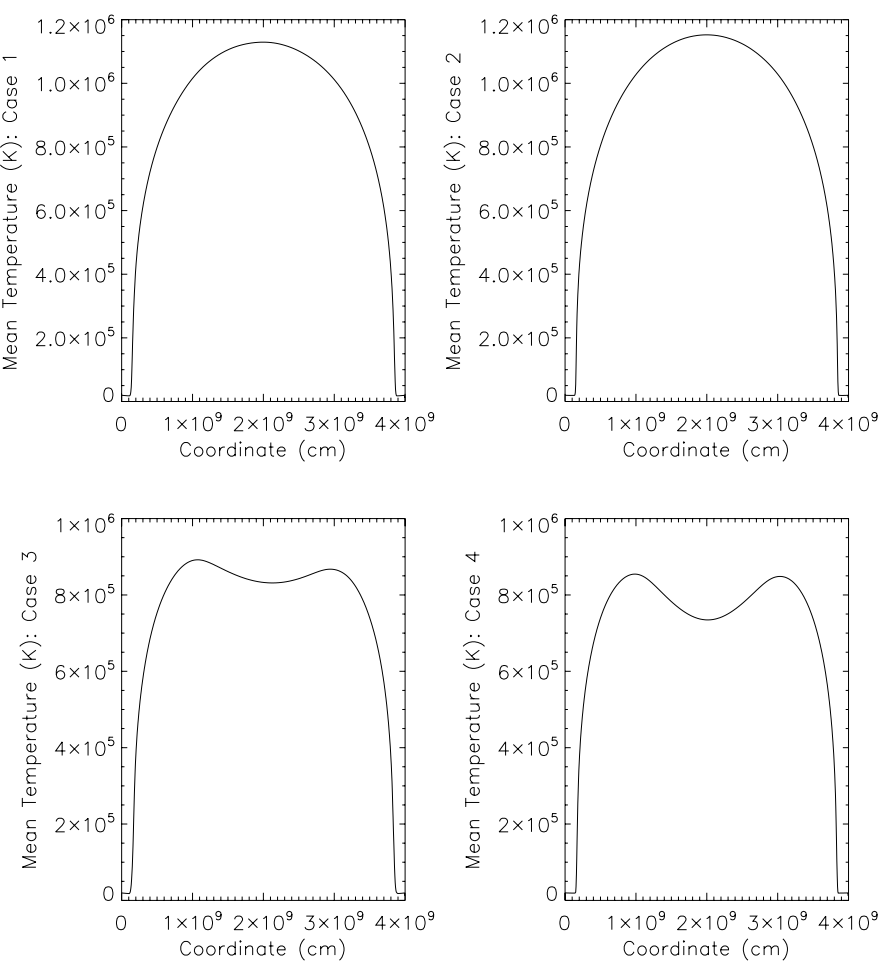

Fig. 2. The mean temperature profile of the loop in four different cases. The top panels characterise uniformly random heating and the bottom panels represent random footpoint heating.

loop, which arise due to the random heating, are correctly resolved. Similar to Eq. (13), we choose the following form for the heating rate:

$$
\begin{aligned}
\mathcal{H}(s, t)= & \mathcal{H}_{0} \sum_{j=1}^{n} H\left(t-t_{j}\right) H\left(t_{j}+\tau_{j}-t\right) \\
& \times \sin \frac{\pi\left(t-t_{j}\right)}{\tau_{j}} \exp \left(-\frac{\left|s-s_{j}\right|}{s_{\mathrm{h}}}\right),
\end{aligned}
$$

where the parameters are defined in the preceding section. The impulsive localised random heating is characterised by small scale lengths. We set $s_{\mathrm{h}}=1 \mathrm{Mm}$ and assume that the minimum height at which heating can take place is $2 \mathrm{Mm}$ above the footpoint base. In the absence of a detailed knowledge of the heating parameters $\tau_{j}, \mathcal{H}_{0}$ and $s_{j}$, we set a maximum duration $\tau_{\max }=10 \mathrm{~s}$ for a single pulse, where $0 \leq \tau_{j} \leq \tau_{\max }$, and consider four different cases:

1. $\mathcal{H}_{0}=0.05 \mathrm{erg} \mathrm{cm}^{-3} \mathrm{~s}^{-1}, n=1000$, uniformly random heating: $0.05 L \leq s_{j} \leq 0.95 L$;

2. $\mathcal{H}_{0}=0.01 \mathrm{erg} \mathrm{cm}^{-3} \mathrm{~s}^{-1}, n=5000$, uniformly random heating: $0.05 L \leq s_{j} \leq 0.95 L$;

3. $\mathcal{H}_{0}=0.05 \mathrm{erg} \mathrm{cm}^{-3} \mathrm{~s}^{-1}, n=1000$, footpoint heating: $0.05 L \leq s_{j} \leq 0.3 L$ and $0.7 L \leq s_{j} \leq 0.95 L$;

4. $\mathcal{H}_{0}=0.01 \mathrm{erg} \mathrm{cm}^{-3} \mathrm{~s}^{-1}, n=5000$, footpoint heating: $0.05 L \leq s_{j} \leq 0.3 L$ and $0.7 L \leq s_{j} \leq 0.95 L$.

The above values are chosen so as to sustain the loop at average temperatures which do not significantly deviate from the initial profile shown in Fig. 1. In the first two cases, it is assumed that the loop is heated randomly along its entire length, i.e., the heating is uniformly random. In dimensional units this corresponds to $2 \mathrm{Mm} \leq s_{j} \leq 38 \mathrm{Mm}$. In the last two cases the 

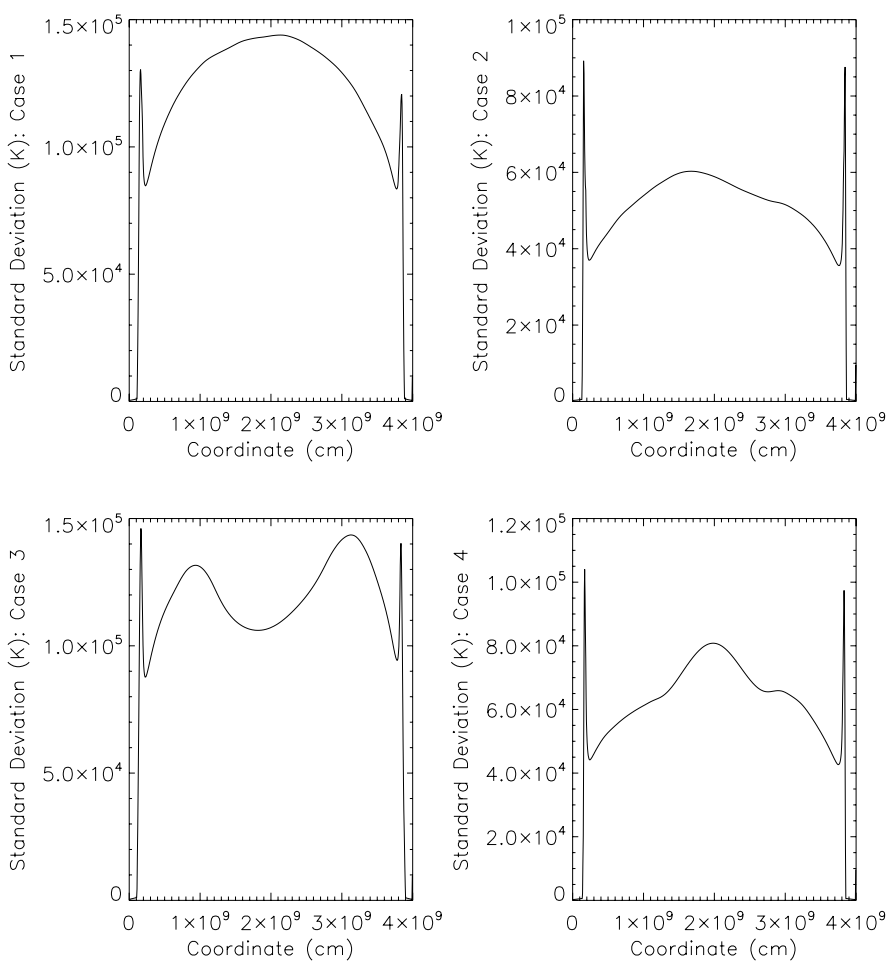

Fig. 3. The standard deviation of temperature in four different cases.

small-scale loop heating takes place near its footpoints. In dimensional units this can be represented as $2 \mathrm{Mm} \leq s_{j} \leq 12 \mathrm{Mm}$ and $28 \mathrm{Mm} \leq s_{j} \leq 38 \mathrm{Mm}$. In the second and fourth cases, the smaller maximum heat input $\mathcal{H}_{0}$ is compensated by larger number of pulses $n$ which occur randomly in time. The mean temperature profile along the loop is plotted in Fig. 2. The mean is taken over the entire duration of the random heating from $t=0 \mathrm{~s}$ to $t=20000 \mathrm{~s}$. The plots show that in the case of uniformly random heating (cases 1 and 2) the mean temperature of the loop reaches higher values than in the case of footpoint heating (cases 3 and 4). Another important quantity characterising the hydrodynamic behaviour of the loop is the standard deviation of temperature defined by:

$\sigma(s)=\sqrt{\frac{1}{20000} \sum_{j=1}^{20000}\left[T_{j}(s)-\bar{T}(s)\right]^{2}}$,

where $T_{j}$ is the temperature at $t=t_{j}$ s and $\bar{T}$ is the mean temperature. The standard deviation measures the average deviation of temperature from its mean. In other words, it shows how spread out the temperature along the loop is. The standard deviation of temperature as a function of coordinate $s$ is plotted in Fig. 3 . Cases 1 and 3, which correspond to more energetic and less frequent pulses, exhibit stronger deviation than cases 2 and 4. However, in cases 2 and 4, the standard deviation in the transition region is more pronounced compared to the rest of the loop. The sharp peaks in the transition region are due to the fact that the random heating results in a dynamically moving transition region. Figure 3 shows that the average temperature variation in the transition region is about $10^{5} \mathrm{~K}$. The corresponding deviation of the velocity in the coronal part of the loop is $10-15 \mathrm{~km} \mathrm{~s}^{-1}$ for cases 1 and 3 , and $5-7 \mathrm{~km} \mathrm{~s}^{-1}$ for cases 2 and 4 .

The mean conductive flux as a function of distance along the loop and measured in erg $\mathrm{cm}^{-2} \mathrm{~s}^{-1}$ is plotted in Fig. 4. The random footpoint heating (bottom panels) leads to a negative flux
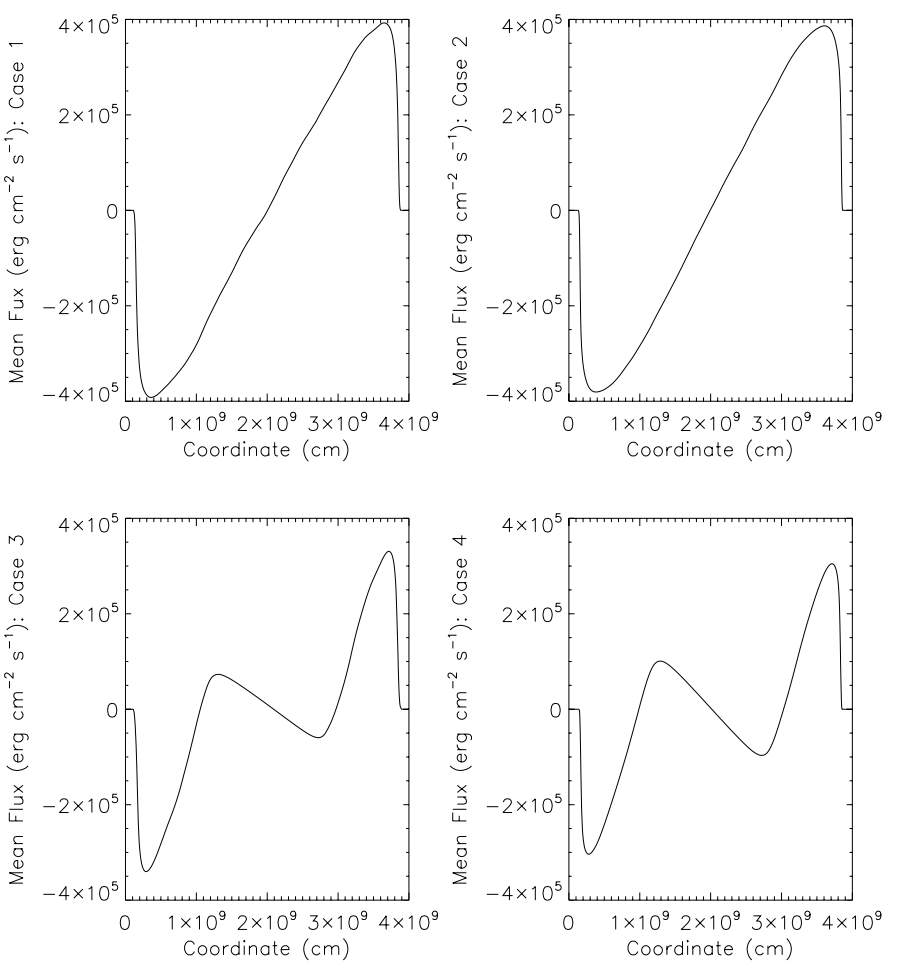

Fig. 4. The mean conductive flux as a function of coordinate $s$ in four different cases.

gradient, i.e., positive conduction around the apex in order to compensate the heat deficit in that region. The mean conductive flux reaches larger values in the case of uniformly random heating (top panels). However, as it can be seen in Fig. 4, the slope of the curve between the peak and the near apex region is steeper in the case of random footpoint heating (bottom panels) than in the case of uniformly random heating (top panels). This implies that in the regions around $s=10^{9} \mathrm{Mm}$ and $s=3 \times 10^{9} \mathrm{Mm}$ the losses due to thermal conduction are greater when random heating is concentrated near the footpoints of the loop.

\section{Expected observational features}

In the present section, the results of the numerical analysis are converted into observable quantities. This allows us to theoretically model possible manifestations of the proposed principle in the observations. The first step in this direction is the derivation of the line profiles.

\subsection{Synthesis of line profiles}

The resonant Ne VIII spectral line, which has a formation temperature of about $8 \times 10^{5} \mathrm{~K}$, is selected for analysis. It is a good representative of the temperature ranges we are interested in. The details of the procedure for the derivation of line profiles are presented in Taroyan et al. (2006). Here we briefly outline the main steps. Positive velocities along the line of sight correspond to red-shift and negative velocities represent blue-shift. The Doppler shift depends on the heliographic position of the loop and the orientation of the loop plane with respect to the slit of the observing instrument. We assume that the observing instrument has typical SUMER (Solar Ultraviolet Measurements of Emitted Radiation spectrometer on board SOHO) characteristics: thus a pixel size of $1^{\prime \prime}$ is taken. The projection of the bulk velocity of the plasma on the line of sight and the corresponding 
Doppler width are calculated. These are used to calculate the line broadening function and the emissivity of the corresponding line. The emissivity is integrated along a loop segment $\left[s_{\min }, s_{\max }\right]$ to obtain the total intensity of the spectral line. The location of the endpoints $s_{\min }, s_{\max }$ along the loop is determined by the heliographic position of the loop and its orientation with respect to the observing instrument. The derived Doppler shift time series for Ne VIII is Fourier analysed to obtain the power spectrum.

\subsection{Examples of power spectra}

In the present section, four different examples are considered in which the loop has different locations and different orientations with respect to the instrument. For simplicity, it is assumed that the loop has zero inclination with respect to the solar normal.

In the first example, the loop is located on the disc centre being oriented transversely to the slit, which crosses the loop between $s_{\min }=10 \mathrm{Mm}$ and

$s_{\max }=\frac{2 L}{\pi} \arcsin \sqrt{\frac{\pi x_{w}}{2 L}+\sin ^{2}\left(\frac{\pi s_{\min }}{2 L}\right)}$,

where $x_{w}=0.7 \mathrm{Mm}$ corresponds to the size of a single pixel. In Eq. (23), $s_{\max }$ is determined by the requirement that the projection of the loop segment $\left[s_{\min }, s_{\max }\right]$ on the plane normal to the line of sight should have a length corresponding to a pixel size of $1^{\prime \prime}$. Within the segment $\left[s_{\min }, s_{\max }\right]$, there is a nonzero component of the bulk velocity on the line of sight which allows us to analyse the Doppler shift time series. The resulting power spectra for four different cases considered in Sect. 3 are plotted in Fig. 5.

The second example corresponds to the case when the loop is located on the solar limb. The loop plane is transverse to the slit and the footpoint baseline is oriented along the line of sight. The slit crosses the loop at the apex, so that there are plasma motions along the line of sight and Doppler shifts can be measured. The coordinate $s$ changes between

$s_{\min }=\frac{L}{\pi} \arcsin \left(1-\frac{\pi x_{w}}{L}\right)$ and $s_{\max }=L-\frac{L}{\pi} \arcsin \left(1-\frac{\pi x_{w}}{L}\right)$,

where $x_{w}=0.7 \mathrm{Mm}$. The power spectrum corresponding to this example is shown in Fig. 6.

In the third example, the loop is located on the disc centre, similar to the first example. However, the loop is oriented along the slit. The coordinate $s$ varies between $s_{\min }=0$ and $s_{\max }=L$, i.e., the intensity is integrated along the entire length of the loop. The corresponding power spectrum is shown in Fig. 7.

The fourth example represents the situation when the loop is on the limb, similar to the second example. However, the loop plane is now aligned with the slit and the footpoint baseline is directed along the line of sight. The emission is therefore integrated along the loop from $s_{\min }=0$ to $s_{\max }=L$. The power spectrum for this case is plotted in Fig. 8.

In addition to the Fourier spectra, the results are presented in terms of wavelet spectra. Three representative cases are selected. The method proposed by Torrence \& Compo (1998) is applied. Figures 9-11 show the results of the wavelet analysis. In Fig. 9, the heating is uniformly random, the loop is located on the disc centre and the slit is transversely oriented. This corresponds to Case 1 in Fig. 5. Similarly, Fig. 10 corresponds to Case 4 in Figs. 5 and 11 corresponds to Case 4 in Fig. 8. The Doppler shift time series measured in $\mathrm{km} \mathrm{s}^{-1}$ is plotted in the top panel (a). In Figs. 9-11, the bottom left panel (b) displays the wavelet power
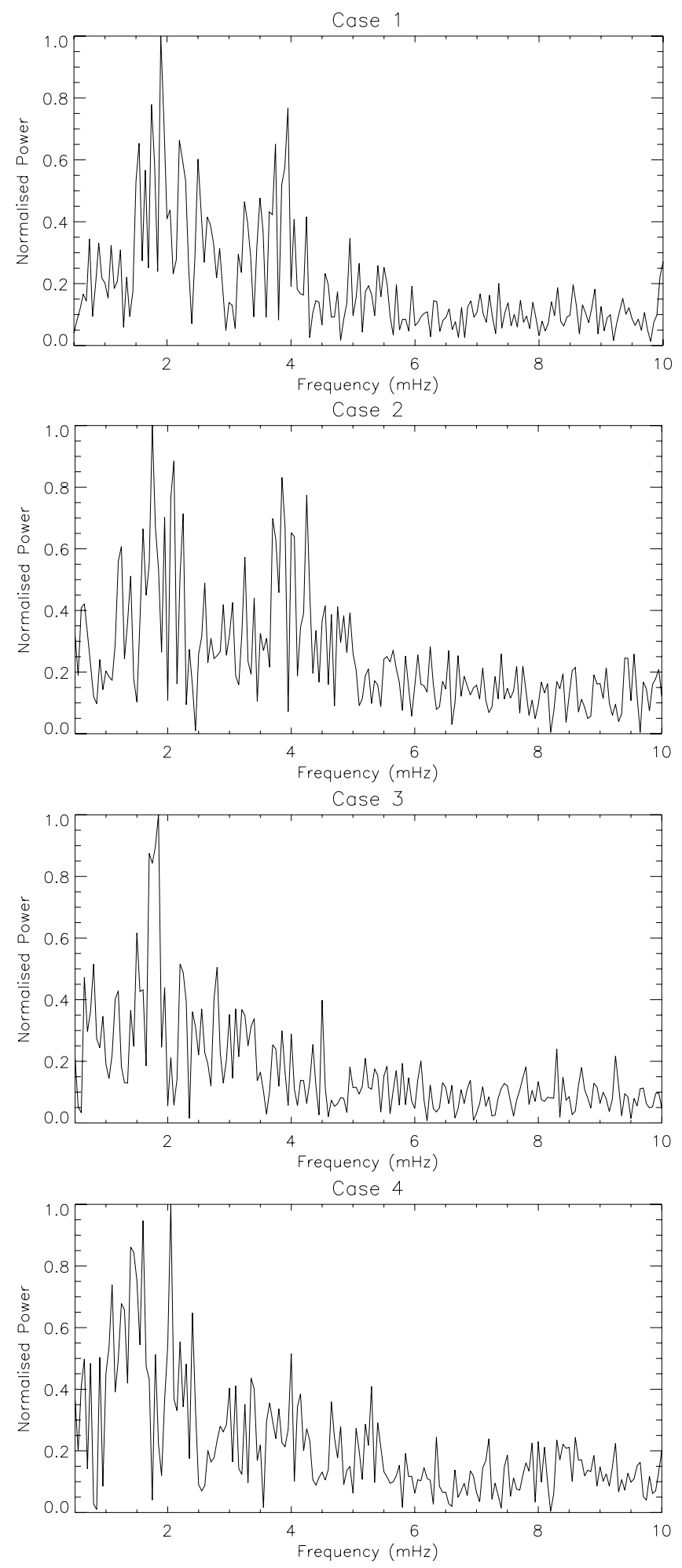

Fig. 5. Power spectrum of the Doppler shift time series for Ne VIII. The loop is situated on the disc centre and its orientation is transverse to the slit. The slit crosses the loop at about $s \approx 11 \mathrm{Mm}$.

spectrum. The right panel (c) represents the global wavelet spectrum (measured in $\mathrm{km}^{2} \mathrm{~s}^{-2}$ ) which is the sum of the wavelet power over time at each oscillation frequency. The red (blue) regions correspond to high (low) power. The cross-hatched region indicates the cone of influence, where edge effects become important. In the wavelet spectrum diagram (b), regions with $90 \%$ 

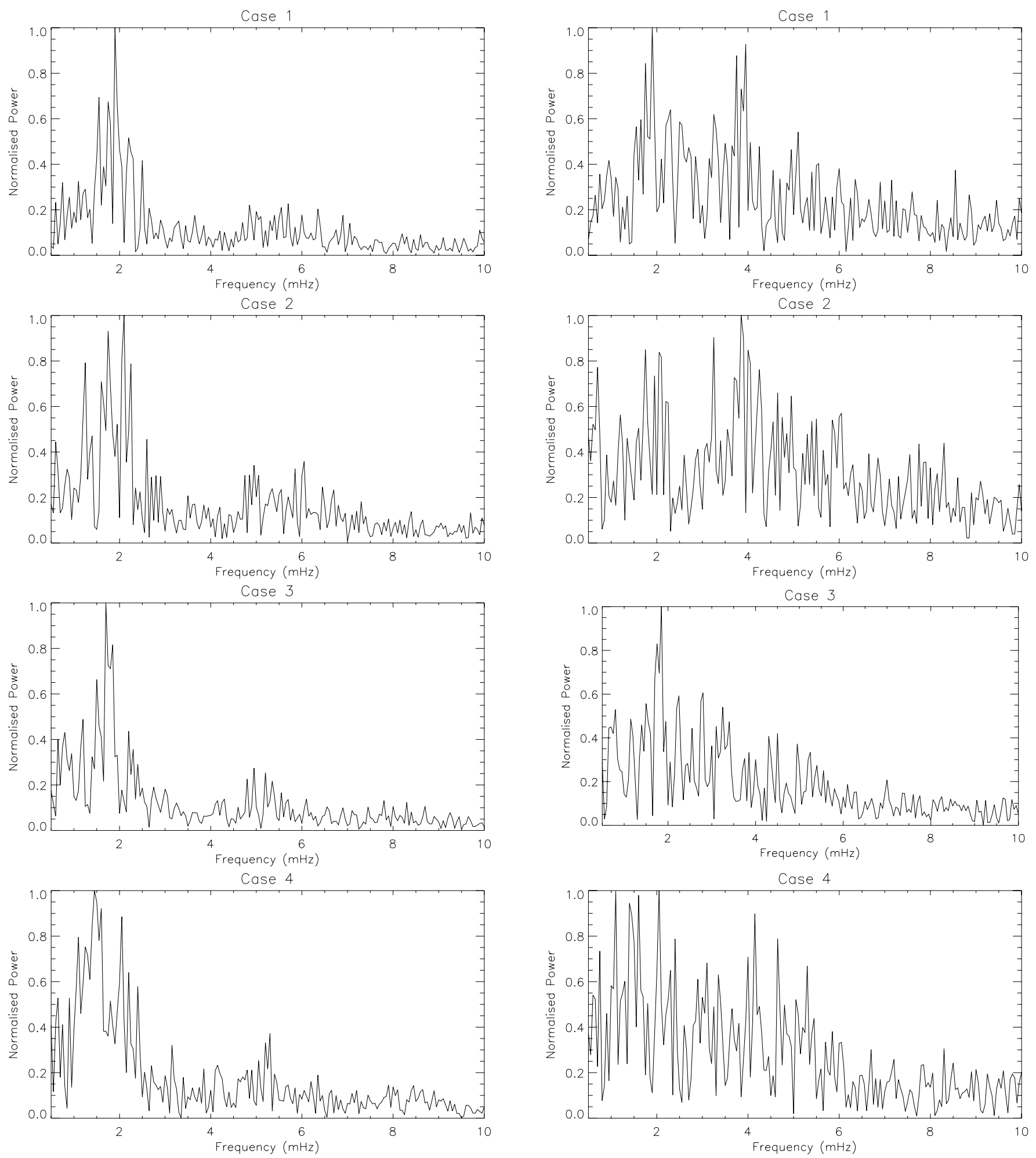

Fig. 6. Power spectrum of the Doppler shift time series for Ne VIII. The loop is situated on the limb and the slit is transversely oriented, crossing the loop at the apex.

significance level are outlined in black. In the global wavelet diagram, the dotted lines indicate $99 \%$ significance level.

\subsection{Discussion}

One can immediately notice in Figs. 5-8 the presence of noise which is caused by the added effects of nonlinearity, thermal

Fig. 7. Power spectrum of the Doppler shift time series for Ne VIII. The loop is situated on the disc centre and the slit is aligned with the loop. The intensity is integrated along the entire length of the loop.

conduction and radiation. However, the power spectra displayed in Figs. 5-8 always show the presence of peaks at around $2 \mathrm{mHz}$ regardless of how the heating occurs. Each peak often represents small clusters of peaks. This can be explained by large variations of temperature in time. The horizontal axis represents frequency defined by $v=1 / P$, where $P$ is the period. The peak corresponds to the frequency of the fundamental mode with $v \approx \bar{c}_{\mathrm{s}} / 2 L$, where 

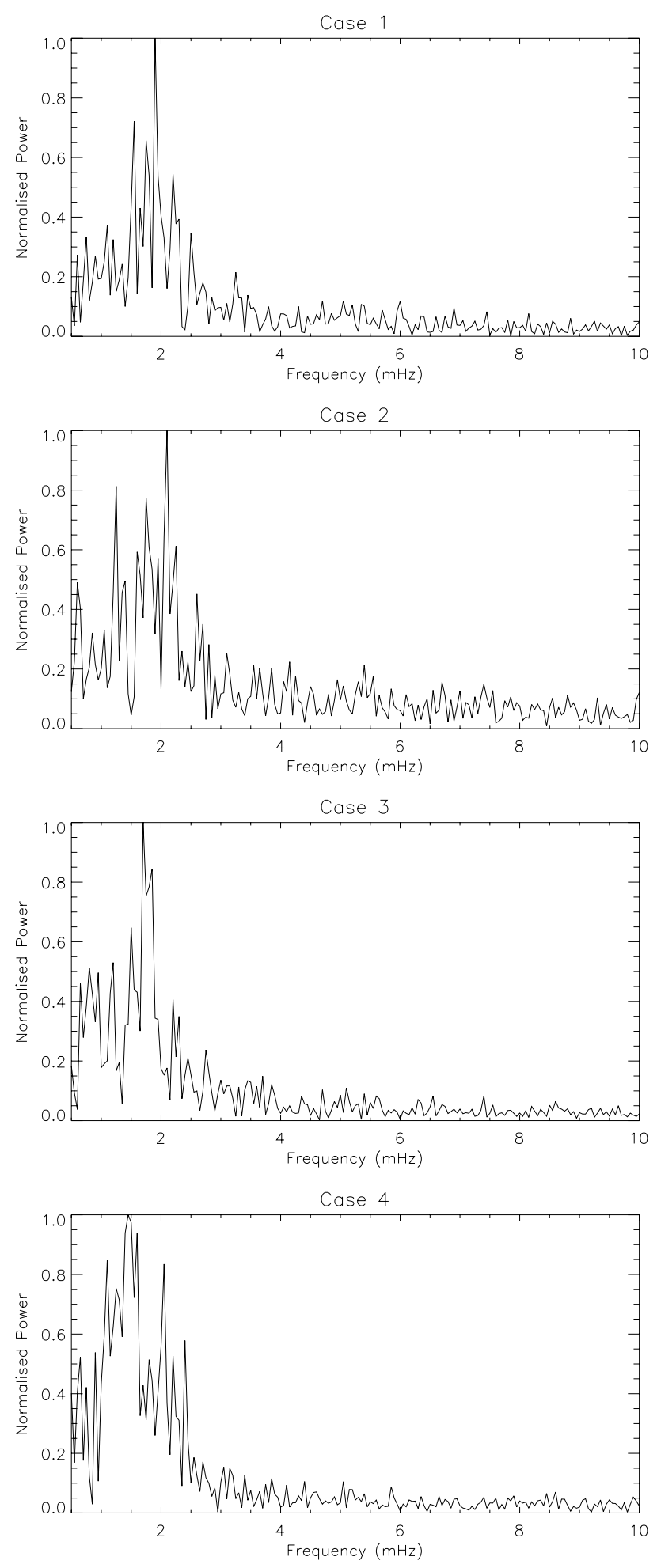

Fig. 8. Power spectrum of the Doppler shift time series for Ne VIII. The loop is situated on the limb and the slit is aligned with the loop plane. The intensity is integrated along the entire length of the loop.

$\bar{c}_{\mathrm{s}}$ is the average sound speed inside the loop. The sound speed is determined by the temperature and, therefore, the power peak could be used to diagnose the average temperature of the loop. The peak corresponding to the fundamental mode, as a rule, is the most prominent. The second high peak with a higher frequency, seen in the upper two panels of Figs. 5 and 7, corresponds to the first harmonic. It has a frequency which is the

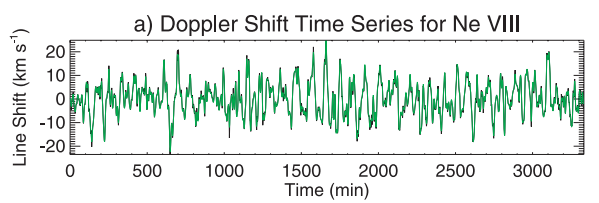

WAVELET ANALYSIS Case 1: Disc Centre, Transverse Orientation
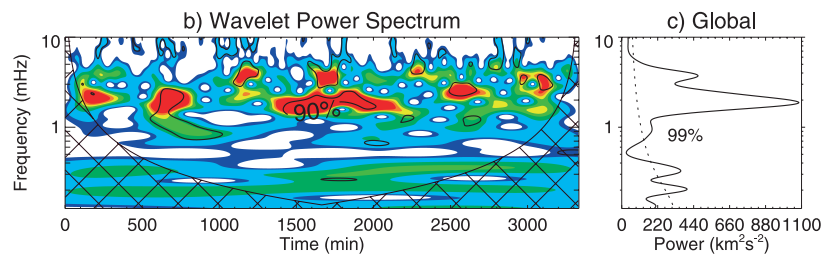

Fig. 9. Wavelet analysis results corresponding to Case 1 in Fig. 5. The top panel a) shows the original Doppler shift time series for Ne VIII. The bottom left panel b) displays the wavelet power spectrum. The red (blue) colour represents high (low) power. The bottom right panel c) is the global wavelet spectrum. In the wavelet spectrum diagram b), regions with $90 \%$ significance level are outlined in black. In the global wavelet diagram, the dotted lines indicate $99 \%$ significance level.

double of the fundamental mode frequency, as one would expect. The peak corresponding to the first harmonic also appears in the global wavelet spectrum in Fig. 9. This peak, however, is much broader and shorter in the lower two panels of Fig. 5 despite the fact that the slit crosses the loop at a segment where the amplitude of the first harmonic is expected to be high compared to the rest of the loop. In fact, the wavelet analysis presented in Fig. 10 shows that the second power peak corresponding to the first harmonic is flattened out in the global wavelet spectrum. This could be explained in the following way: the lower two panels in Fig. 5 represent cases in which the random heating is concentrated near the footpoints. According to Fig. 4, these cases correspond to larger gradients of the conductive flux around $s=L / 4$ and $s=3 L / 4$ where the amplitude of the first harmonic has maxima. This leads to stronger damping by thermal conduction and the expected peaks corresponding to the first harmonic are flattened out. The situation is similar in Fig. 7 where the intensity along the entire loop is considered. Here, however, one can see some power for the first harmonic in the bottom panel. The peak corresponding to the first harmonic is totally absent in Figs. 6, 8, and 11c. The absence of the second peak in Fig. 6 can be explained by the fact that the slit crosses the loop at the apex where the first harmonic has a node. In the fourth example, the slit is aligned with the loop plane and, therefore, signals from the two legs of the loop with opposite Doppler shifts cancel each other. This explains the absence of peaks at around $4 \mathrm{mHz}$ in Figs. 8 and $11 \mathrm{c}$. Therefore, one could analyse the power spectra in order to determine whether the loops are heated uniformly or near their footpoints.

Another important feature seen in Figs. 9-11 is the difference between cases 1, 3 (more energetic and less frequent events) and cases 2, 4 (less energetic and more frequent events). The latter two cases correspond to the bottom right panels in Figs. 10 and 11. The maximum power in these panels is small compared with the maximum power seen in Fig. 9. A comparison of the top panels (a) in Figs. 9-11 shows that the velocity amplitudes are smaller in the case of less energetic and more frequent events (Cases 2, 4). The smaller amplitudes lead to shorter peaks in Figs. 10c and 11c. Therefore, the power spectra could be potentially useful in estimating the average amount of energy involved in a single heating event. 


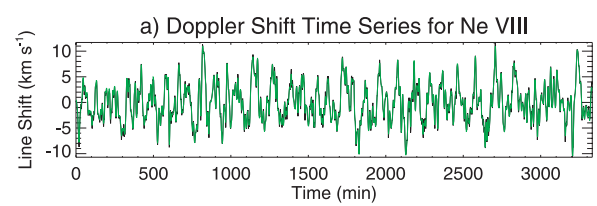

WAVELET ANALYSIS Case 4: Disc Centre, Transverse Orientation

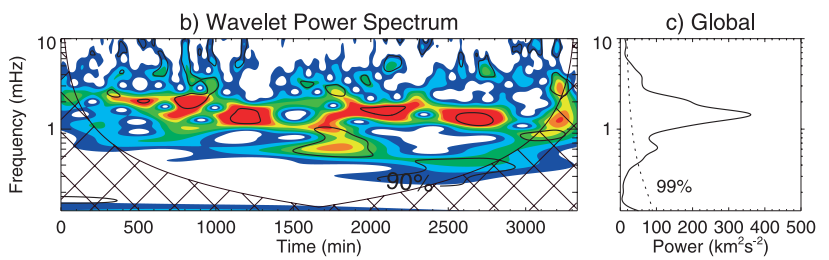

Fig. 10. Same as in Fig. 9 except that the wavelet analysis results correspond to Case 4 in Fig. 5.

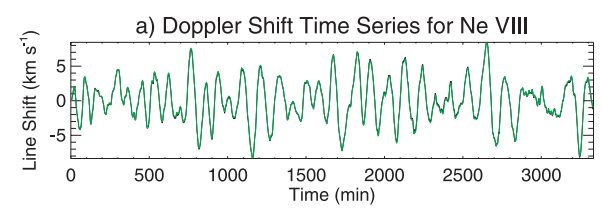

WAVELET ANALYSIS Case 4: Solar Limb, Parallel Orientation

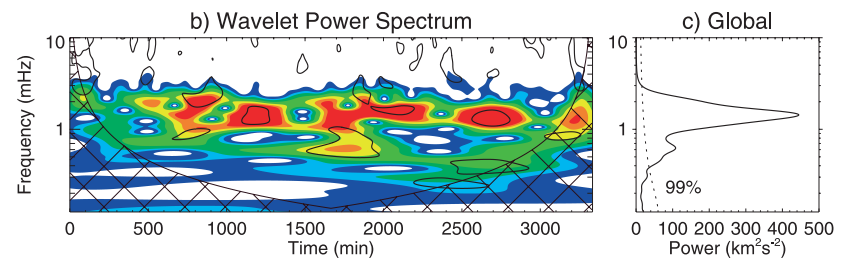

Fig. 11. Same as in Fig. 9 except that the wavelet analysis results correspond to Case 4 in Fig. 8.

It is well-known that the loops seen in high resolution TRACE images appear to have multistrand structuring. The understanding of such strands is a major issue in solar coronal physics. In this respect, the proposed method could be useful too. Each strand would have a peak and possibly a corresponding overtone in the power spectrum. By studying these peaks, one could, in principle, determine the way in which the individual strands are heated and their average temperatures. However, this remains a speculation. Combined theoretical and observational work is needed in order to find out the extent of applicability of the proposed method.

\section{Conclusions}

1. A new diagnostic method for randomly heated loops is proposed.

2. The results of the analytical study show that for a linear ideal 1D loop model heated by random pulses there are an infinite number of peaks in the velocity power spectrum which correspond to the frequencies of standing waves. The peaks should become taller and narrower as the duration of the observations becomes longer.

3. The inclusion of radiative losses, thermal conduction and nonlinearity introduces noise in the power spectrum.

4. The most prominent peak corresponding to the fundamental mode is always present regardless of the random heating function and the heliographic position of the loop. This peak could therefore be used to determine the average temperature of the plasma inside the loop.
5. The peaks corresponding to the first harmonic only appear in the case of uniformly random heating. Loops heated near their footpoints only display the peak corresponding to the fundamental mode. The physical mechanism responsible for this phenomenon is explained. The power spectrum analysis could be used to establish the spatial distribution of the random heating.

6. The power spectra are sensitive to the temporal distribution of heating and, as a result, they could be useful in estimating the average amount of energy involved in a single heating event.

7. The power spectrum analysis could be applied to study the multistrand structuring of the loops.

Acknowledgements. The simulations were run on White Rose Grid (Iceberg node at Sheffield University). Y.T. is grateful to PPARC for financial support. R.E. acknowledges M. Kéray for patient encouragement and NSF, Hungary (OTKA, ref. No. TO43741). Research at Armagh Observatory is grant-aided by the N. Ireland Dept. of Culture, Arts and Leisure. S.J.B. is grateful to PPARC for their support through the award of a Post-Doctoral Fellowship. Wavelet software was provided by C. Torrence and G. Compo, and is available at http://paos.colorado.edu/research/wavelets.

\section{Appendix A: Coefficients in Eqs. (12) and (15)}

$$
\begin{aligned}
C_{j k}=\frac{1}{\mathrm{i} k} & \sin \left(\frac{\omega_{k} s_{\mathrm{h}}}{c_{\mathrm{s}}}\right) \cos \left(\frac{\omega_{k} s_{j}}{c_{\mathrm{s}}}\right) \sin \left(\frac{\omega_{k} s}{c_{\mathrm{s}}}\right) \\
& \times \exp \left[\mathrm{i} \omega_{k} t_{j}+\mathrm{i}\left(\omega-\omega_{k}\right) \frac{t_{\mathrm{d}}+t_{j}}{2}\right], \\
C_{1 j k}=\frac{1}{k^{2}} \sin \left(\frac{\omega_{k} s_{\mathrm{h}}}{c_{\mathrm{s}}}\right) & \cos \left(\frac{\omega_{k} s_{j}}{c_{\mathrm{s}}}\right) \sin \left(\frac{\omega_{k} s}{c_{\mathrm{s}}}\right) \\
& \times 2 \exp \left[\mathrm{i} \omega t_{j}+i \omega \frac{\tau_{j}}{2}\right]
\end{aligned}
$$

for positive $k$ and 0 for negative $k$,

$$
\begin{array}{r}
C_{2 j k}=-\frac{1}{k^{2}} \sin \left(\frac{\omega_{k} s_{\mathrm{h}}}{c_{\mathrm{s}}}\right) \cos \left(\frac{\omega_{k} s_{j}}{c_{\mathrm{s}}}\right) \sin \left(\frac{\omega_{k} s}{c_{\mathrm{s}}}\right) \\
\quad \times \exp \left[\mathrm{i} \omega t_{j}+\mathrm{i}\left(\omega-\omega_{k}\right) \frac{t_{\mathrm{d}}-t_{j}}{2}\right], \\
C_{3 j k}=\frac{1}{k^{2}} \sin \left(\frac{\omega_{k} s_{\mathrm{h}}}{c_{\mathrm{s}}}\right) \cos \left(\frac{\omega_{k} s_{j}}{c_{\mathrm{s}}}\right) \sin \left(\frac{\omega_{k} s}{c_{\mathrm{s}}}\right) \\
\times \exp \left[\mathrm{i} \omega\left(t_{j}+\tau_{j}\right)+\mathrm{i}\left(\omega-\omega_{k}\right) \frac{t_{\mathrm{d}}-t_{j}-\tau_{j}}{2}\right]
\end{array}
$$

\section{References}

Aschwanden, M. J., 2001, A\&A, 560, 1035

Aschwanden, M. J., Fletcher, L., Schrijver, C. J., \& Alexander, D. 1999, ApJ, 520,880

Bradshaw S. J., \& Mason H. E., 2003, A\&A, 407, 1127

Brković, A., Landi, E., Landini, M., Rüedi, I., \& Solanki, S. 2002, A\&A, 383, 661

Chae, J., Park, Y. D., Moon, Y.-J., Wang, H., \& Yun, H. S. 2002, ApJ, 567, L159 De Moortel, 2005, Royal Soc. London Trans. Ser. A, 363, 2743

De Moortel, I., Ireland, J., \& Walsh, R.W. 2000, A\&A, 355, L23

De Pontieu, B., Erdélyi, R., \& De Moortel, I. 2005, ApJ, 624, L61

De Pontieu, B., Erdélyi, R., \& James, S. P. 2004, Nature, 430, 546

Del Zanna, G., Mason, H. E. 2003, A\&A, 406, 1089

Doyle, J. G., Popescu, M. D., \& Taroyan, Y., 2006a, A\&A 446, 327

Doyle, J. G., Taroyan, Y., Ishak, B., Madjarska, M. S., \& Bradshaw, S. J., 2006b, A\&A, 452, 1075

Duffy, D. G. 2001, Green's functions with applications, Studies in Advanced Mathematics, (Chapman \& Hall/CRC)

Einaudi, G., \& Velli, M. 1999, Phys. Plasmas, 6, 4146 
Landi, E., \& Landini, M. 2004, ApJ, 608, 1133

Landi, E., \& Landini, M. 2005, ApJ, 618, 1039

Martens, P. C. H., Cirtain, J. W., \& Schmelz, J. T. 2002, ApJ, 577, L115

Mendoza-Briceño, C. A., \& Erdélyi, R., 2006, ApJ, 648, 722

Moriyasu, S., Kudoh, T., Yokoyama, T., \& Shibata, K. 2004, ApJ, 601, L107

Musielak, Z. E., \& Ulmschneider, P. 2001, A\&A, 370, 541

Nakariakov, V. M., Ofman, L., DeLuca, E. E., Roberts, B., \& Davila, J. M. 1999, Science, 285,862

Nakariakov, V. M., \& Verwichte, E. 2005, Living Rev. Sol. Phys. 2, 3

Parker, E. N. 1988, ApJ, 330, 474

Priest, E. R., Foley, C. R., Heyvaerts, J., Arber, T. D. et al. , 1998, Nature, 393, 545

Reale F., 2002, ApJ, 580, 566
Rosner, R., Golub, L., Coppi, B., \& Vaiana, G. S. 1978, ApJ, 222, 317

Ruderman, M. S., \& Roberts, B. 2002, ApJ, 577, 475

Schmelz, J. T., \& Martens, P. C. H. 2006, ApJ, 636, L49

Schmelz, J. T., Scopes, R. T., \& Cirtain, J. W. 2002, Adv. Space Res., 30, 507

Taroyan Y., Erdélyi, R., Bradshaw, S. J., \& Doyle, J. G. 2005, A\&A, 438, 713

Taroyan Y., Bradshaw, S. J., \& Doyle, J. G. 2006, A\&A, 446, 315

Testa, P., Peres, G., Reale, F., \& Orlando, S. 2002, ApJ, 580, 1159

Torrence, C., \& Compo, G. P., 1998, Bull. Amer. Meteor. Soc., 79, 61

Ugarte-Urra, I., Doyle, J. G., Walsh, R. W., \& Madjarska, M. S., 2005, A\&A, 439,351

Wang, T. J., Solanki, S. K., Curdt, W., Innes, D. E., \& Dammasch, I. E. 2002, ApJ, 574, L101

Wang, T. J., Solanki, S. K., Innes, D. E., \& Curdt, W. 2005, A\&A, 435, 753 